\title{
THE LOCAL UNIVERSE AS SEEN IN THE FAR-INFRARED AND FAR-ULTRAVIOLET: A GLOBAL POINT OF VIEW OF THE LOCAL RECENT STAR FORMATION
}

\author{
V. Buat, ${ }^{1}$ T. T. Takeuchi, ${ }^{1,2}$ J. Iglesias-Páramo, ${ }^{3}$ C. K. Xu,${ }^{4}$ D. Burgarella, ${ }^{1}$ A. Boselli,,${ }^{1}$ T. Barlow, ${ }^{2}$ \\ L. Bianchi, ${ }^{5}$ J. Donas, ${ }^{1}$ K. Forster, ${ }^{4}$ P. G. Friedman, ${ }^{4}$ T. M. Heckman, ${ }^{6}$ Y.-W. Lee, ${ }^{7}$ B. F. Madore, ${ }^{8,9}$ \\ D. C. Martin, ${ }^{4}$ B. Milliard, ${ }^{1}$ P. Morissey, ${ }^{4}$ S. Neff, ${ }^{10}$ M. Rich, ${ }^{11}$ D. Schiminovich, ${ }^{12}$ M. Seibert, ${ }^{4}$ \\ T. Small, ${ }^{4}$ A. S. Szalay, ${ }^{6}$ B. Welsh, ${ }^{13}$ T. Wyder, ${ }^{4}$ and S. K. Y ${ }^{7}$ \\ Received 2006 June 9; accepted 2006 September 25
}

\begin{abstract}
We select far-infrared (FIR: $60 \mu \mathrm{m}$ ) and far-ultraviolet (FUV: $530 \AA$ ) samples of nearby galaxies in order to discuss the biases encountered by monochromatic surveys (FIR or FUV). Very different volumes are sampled by each selection, and much care is taken to apply volume corrections to all the analyses. The distributions of the bolometric luminosity of young stars are compared for both samples: they are found to be consistent with each other for galaxies of intermediate luminosities, but some differences are found for high $\left(>5 \times 10^{10} L_{\odot}\right)$ luminosities. The shallowness of the IRAS survey prevents us from securing a comparison at low luminosities $\left(<2 \times 10^{9} L_{\odot}\right)$. The ratio of the total infrared (TIR) luminosity to the FUV luminosity is found to increase with the bolometric luminosity in a similar way for both samples up to $5 \times 10^{10} L_{\odot}$. Brighter galaxies are found to have a different behavior according to their selection: the $L_{\mathrm{TIR}} / L_{\mathrm{FUV}}$ ratio of the FUV-selected galaxies brighter than $5 \times 10^{10} L_{\odot}$ reaches a plateau, whereas $L_{\mathrm{TIR}} / L_{\mathrm{FUV}}$ continues to increase with the luminosity of bright galaxies selected in FIR. The volume-averaged specific star formation rate (SFR per unit galaxy stellar mass, SSFR) is found to decrease toward massive galaxies within each selection. The mean values of the SSFR are found to be larger than those measured for optical and NIR-selected samples over the whole mass range for the FIR selection, and for masses larger than $10^{10} M_{\odot}$ for the FUV selection. Luminous and massive galaxies selected in FIR appear as active as galaxies with similar characteristics detected at $z \sim 0.7$.
\end{abstract}

Subject headings: dust, extinction — galaxies: photometry — galaxies: stellar content — infrared: galaxies — ultraviolet: galaxies

Online material: color figures, machine-readable tables

\section{INTRODUCTION}

Many of the most recent galaxy surveys have attempted to gain a better understanding of the evolution of the star formation rate (SFR) with time and environment. Because of spectral redshifting, deep (high-redshift) optical surveys in fact sample the far-ultraviolet (FUV) rest-frame emission of the target galaxies. As a consequence, numerous measurements of the star formation activity of galaxies as a function of redshift $(z)$ are based on restframe FUV data obtained from imaging and spectroscopic sur-

\footnotetext{
${ }^{1}$ Laboratoire d'Astrophysique de Marseille, Marseille 13012, France.

2 Astronomical Institute, Tohoku University, Aoba, Aramaki, Aoba-ku, Sendai 980-8578, Japan.

3 Instituto de Astrofísica de Andalucía, CSIC, 18008 Granada, Spain.

${ }^{4}$ California Institute of Technology, MC 405-47, 1200 East California Boulevard, Pasadena, CA 91125.

${ }_{5}$ Center for Astrophysical Sciences, The Johns Hopkins University, 3400 North Charles Street, Baltimore, MD 21218.

6 Department of Physics and Astronomy, The Johns Hopkins University, Homewood Campus, Baltimore, MD 21218.

7 Center for Space Astrophysics, Yonsei University, Seoul 120-749, Korea.

8 Observatories of the Carnegie Institution of Washington, 813 Santa Barbara Street, Pasadena, CA 91101.

9 NASA/IPAC Extragalactic Database, California Institute of Technology, MC 100-22, 770 South Wilson Avenue, Pasadena, CA 91125.

${ }^{10}$ Laboratory for Astronomy and Solar Physics, NASA Goddard Space Flight Center, Greenbelt, MD 20771.

11 Department of Physics and Astronomy, University of California, Los Angeles, CA 90095.

12 Department of Astronomy, Columbia University, New York, NY 10027.

13 Space Sciences Laboratory, University of California at Berkeley, Berkeley, CA 94720.
}

veys: at low $z$ (e.g., Lilly et al. 1996; Schiminovich et al. 2005; Baldry et al. 2005) or at higher $z$, using dropout selection techniques (e.g., Steidel et al. 1999; Bunker et al. 2004; Ouchi et al. 2004; Giavalisco et al. 2004). However, the attenuation of the FUV light by interstellar dust is a major issue in the derivation of quantitative SFR from the FUV even at low $z$ (e.g., Buat et al. 2005; Seibert et al. 2005; Cortese et al. 2006).

Recent infrared surveys (e.g., Flores et al. 1999; Le Floc'h et al. 2005; Pérez-González et al. 2005) have also contributed significantly to the study of the star formation history in the universe (e.g., Rowan-Robinson 2001; Takeuchi et al. 2001a, 2001b; Lagache et al. 2003): the far-infrared (FIR) emission from the dust heated by hot stars is, by definition, not affected by dust attenuation. However, FIR emission has its own drawbacks: the calibration of the dust emission into a quantitative SFR usually relies on the strong assumption that most of the dust heating is due to young stars and that all the light from these stars is absorbed by dust and re-emitted in FIR (e.g., Kennicutt 1998). This is clearly an oversimplification: most galaxies are seen to emit in the FUV and optical, and as a consequence all the light emitted by young stars cannot have been absorbed by dust. Moreover, substantial dust heating by older stars cannot be ruled out for all galaxies, and more complex calibrations have to be undertaken (Buat \& Xu 1996; Bell 2003; Hirashita et al. 2003; IglesiasPáramo et al. 2006). Another issue is that infrared observations are carried out at one or a few wavelengths (in the mid and/or far-infrared), whereas it is the total infrared (TIR) emission that is required for a star formation rate calculation. Unfortunately, the TIR emission is derived by extrapolation from only a few measured fluxes using models, and the correction factors range from 
2 to 3 when observations are made in the far-infrared, and up to 8-10 when only mid-infrared data are available (e.g., Takeuchi et al. 2005b; Le Floc'h et al. 2005).

The ultraviolet and the FIR emission are each strongly linked to alternate manifestations of the recent star formation rate: the "transparent" one in FUV and the "hidden" one in FIR. Obviously, a very promising way to proceed would be to combine both wavelengths to perform a more inclusive and multiwavelength analysis of the current star formation in the universe.

What do we know today about the FUV and FIR universe? From a global point of view, the recent observations conducted by the Spitzer Space Telescope and the Galaxy Evolution Explorer (GALEX) have provided insight into the TIR and FUV luminosity functions and densities from $z=0$ to 1 (Schiminovich et al. 2005; Le Floc'h et al. 2005; Pérez-González et al. 2005; Arnouts et al. 2005). The shapes of the luminosity functions in both wavelength ranges are found to be very different (Takeuchi et al. 2005a), as previously emphasized by Buat \& Burgarella (1998). The evolution of the luminosity functions and the derived star formation densities have been studied in the FUV (Schiminovich et al. 2005) and at infrared wavelengths (Le Floc'h et al. 2005; Pérez-González et al. 2005): a strong evolution was found at both wavelengths, with a net increase of the luminosity density from $z=0$ to 1 . Nevertheless, the evolution appears to be stronger in the FIR than in the FUV, implying a global increase of the dust attenuation from $z=0$ to 1 by $\sim 1$ mag (Takeuchi et al. 2005a). Such an increase might be explained, at least qualitatively, by the larger fraction of bright galaxies at high redshift, combined with the known positive correlation of the dust attenuation with the absolute luminosity of the host galaxies (e.g., Wang \& Heckman 1996; Buat \& Burgarella 1998; Hopkins et al. 2001; Martin et al. 2005). In a recent analysis of SWIRE GALEX data Xu et al. (2007) found no significant differences between the FIR-to-FUV flux ratios of star-forming galaxies at $z=0.6$ and their local counterparts of similar SFR. They argued that the evolution of the dust attenuation with redshift is primarily due to the SFR evolution and to the strong dependence of the attenuation on SFR itself.

Indeed, most recent studies based on large surveys aim at better understanding of which galaxies are at the origin of the variation of the star formation density with $z$, and especially its decrease from $z \sim 1$ to 0 , which is seen at almost all wavelengths (e.g., Hopkins \& Beacom 2006 and references therein). From the UV-optical side, almost all recent surveys have found a strong increase in luminosity and/or space density of late type blue galaxies, although some discrepancies have been noted. Differences in the definition of galaxy types from one study to another make a direct comparison of the results problematic (e.g., de Lapparent et al. 2004; Wolf et al. 2005 and references therein). From the IR side, recent results from the Spitzer Space Telescope seem to attribute the general decrease of the star formation density to a decrease in the SFR in massive spirals (Bell et al. 2005) without strong interactions. Studies, both in the IR and in the optical, suggest a minimal role of strong mergers in the evolution of the star formation density from $z=1$ to 0 (Lotz et al. 2006; Bell et al. 2005).

Connecting from low- to high- $z$ that which is seen in the restframe FIR or in the FUV is a new challenge. Do we see the same galaxy populations in the FUV and FIR evolving as a whole and appearing differently in FUV and FIR, or must we invoke subpopulations of galaxies evolving independently with $z$ and/or being detected only at one wavelength, FUV or FIR? This is a crucial question: if we are observing the same populations in both wavelength ranges, then, with some justification, we can try to predict the total star formation from single-band surveys (assum- ing some relevant corrections). But if other populations are present, it would appear to be impossible to reconstruct all of the star formation activity from a single-band survey.

The first step consists of obtaining a reliable reference data set in the local universe. Thanks to GALEX, large samples of nearby galaxies observed in the FUV are now available. Making use of the existing IR surveys, we can then build robust reference samples of galaxies selected in the FUV and FIR which are suitable for comparison. The aim of this paper is to take advantage of the GALEX shallow survey to build large samples of nearby galaxies selected in the FUV (or in the FIR by IRAS) and to use these samples to analyze the selection biases and the consistency of the FIR and FUV LFs at $z=0$. We can then build large reference samples of galaxies selected at $60 \mu \mathrm{m}$, for instance, in such a way as to allow a very good detection rate at $1530 \AA$ and vice versa ( 2 2). In $\S 3$ we emphasize the intrinsic differences existing between a FIR and FUV selection. The relative contributions of the TIR and FUV emissions to the luminosity of the young stellar populations in galaxies is assessed in $\S 4$ using a cross-comparison of the luminosity functions in both samples; bolometric luminosity functions are then built to check if we can indeed see all the galaxies at a single wavelength. In $\S 5$ we discuss the star formation activity as a function of the stellar mass in both samples through an analysis of the specific star formation rate (SSFR; SFR per unit galaxy stellar mass). Section 6 is devoted to the conclusions. This works extends the earlier studies of Martin et al. (2005), Iglesias-Páramo et al. (2006), and Xu et al. (2006), which were based on smaller samples.

Throughout this article we use the cosmological concordance parameters of $H_{0}=72 \mathrm{~km} \mathrm{~s}^{-1} \mathrm{Mpc}^{-1}, \Omega_{M}=0.3$, and $\Omega_{\lambda}=0.7$. All magnitudes are quoted in the $\mathrm{AB}$ system.

\section{THE GALAXY SAMPLES}

\subsection{The FIR-Selected Sample}

The FIR-selected sample was selected from the IRAS PSC $z$ (Saunders et al. 2000). We selected all of the confirmed galaxies (reliability $\geq 50 \%$ in the PSC $z$ ) in the $\sim 3000 \mathrm{deg}^{2}$ covered by the first public release of the GALEX All-Sky Imaging Survey (AIS); 777 sources not contaminated by cirrus (cirrus flag lower than 2) were retrieved over an effective area of $2210 \mathrm{deg}^{2}$. We then extracted FUV images for each of these sources: most of these objects are resolved by $G A L E X$, and we performed the photometry manually for each source, since the GALEX pipeline reduction currently works only for point sources. A detailed description of the photometric process is given in Iglesias-Páramo et al. (2006). Seventy-seven galaxies were not detected in the FUV; 28 of these 77 nondetected sources were located near the edge of the GALEX field, where the image quality is degraded. For the remaining nondetections we adopted an upper limit to the FUV of $20.5 \mathrm{mag}$, corresponding to the $3 \sigma$ detection limit in the AIS (Morrissey et al. 2005). At this point, we were left with 749 galaxies from the $\mathrm{PSC} z$ which had either an FUV measurement or an upper limit.

The lower spatial resolution of IRAS observations sometimes leads to confusion in the selection of the FUV counterpart for a given FIR source. In order to check this we searched for neighbors detected by the Two Micron All Sky Survey (2MASS) or the NRAO VLA Sky Survey (NVSS) within a radius of $1^{\prime}$ around the IRAS position. We individually checked all IRAS sources with several neighbors by superimposing the 2MASS, GALEX, IRAS, and NVSS images. Out of the 749 sources, 63 were considered to be confused. These galaxies were kept for the determination of the FIR LF, but they were not included 
TABLE 1

FIR-Selected SAMPLE

\begin{tabular}{|c|c|c|c|c|c|c|}
\hline $\begin{array}{l}\text { IRAS Name } \\
\text { (1) }\end{array}$ & $\begin{array}{l}F_{60} \\
(\mathrm{Jy}) \\
(2)\end{array}$ & $\begin{array}{l}F_{100} \\
(\mathrm{Jy}) \\
(3)\end{array}$ & $\begin{array}{c}\text { FUV } \\
(\mathrm{mag}) \\
(4)\end{array}$ & $\begin{array}{c}c z \\
\left(\mathrm{~km} \mathrm{~s}^{-1}\right) \\
(5)\end{array}$ & $\begin{array}{c}H \\
(\mathrm{mag}) \\
(6)\end{array}$ & $\begin{array}{c}\text { Type } \\
\text { (7) }\end{array}$ \\
\hline RAS $00003-0747 \ldots$ & 0.65 & 0.69 & 19.75 & 8697 & 12. & $\mathrm{Sab}$ \\
\hline IRAS 00005 & 0.84 & 2.58 & 16.60 & 7271 & 10.53 & $\mathrm{Sbc}$ \\
\hline IRAS $00007+0235 \ldots \ldots$ & 1.08 & 1.38 & $\ldots$ & 27855 & 13.82 & \\
\hline IRAS & 0.96 & 1.41 & 16.65 & 7134 & 11.94 & $\mathrm{Sb}$ \\
\hline IRAS $00025-0722 \ldots \ldots$ & 1.07 & 3.22 & 15.57 & 3765 & 10.53 & $\mathrm{SBb}$ \\
\hline
\end{tabular}

Notes.-Col. (1): IRAS name from the IRAS Point Source Catalog. Col. (2): Flux at $60 \mu \mathrm{m}$. Col. (3): Flux at $100 \mu \mathrm{m}$. Col. (4): FUV magnitude (AB scale) corrected for Galactic extinction (see text). When no value is quoted an upper limit of $20.5 \mathrm{mag}$ is adopted (see text). Col. (5): Radial velocity from the PSC $z$. Col. (6): Total $H$ magnitude from 2MASS. Col. (7): Morphological type from HyperLeda or NED (the values taken from NED are preceded by a period). Table 1 is published in its entirety in the electronic edition of the Supplement. A portion is shown here for guidance regarding its form and content.

in the analysis of the FIR and FUV properties of individual galaxies.

In the end, we were left with a sample of 686 unconfused sources for which an FUV detection (or an upper limit) was available. Twenty-one galaxies are noted in the NASA/IPAC Extragalactic Database (NED) as being active galaxies. This number must be taken as a lower limit since such detailed information in the NED is available for only approximately two-thirds of the sample galaxies. Nevertheless, the contamination of our sample by active galaxies is here estimated to be lower than $1.5 \%$. In order to have reliable distances (as determined from expansion velocities) we considered only the 665 galaxies with velocities $v>1000 \mathrm{~km} \mathrm{~s}^{-1}$. The final sample of 665 sources is presented in Table 1: the FUV fluxes are corrected for foreground Galactic extinction using the Schlegel et al. (1998) dust map and the Cardelli et al. (1989) extinction curve. Seventy-seven of these sources were not detected at $100 \mu \mathrm{m}$; and for these galaxies we estimated the fluxes at $100 \mu \mathrm{m}$ using a mean value of $F_{60} / F_{100}=0.5(\sigma=0.2)$ derived from the FIR galaxies in our sample that were detected at both wavelengths. From 2MASS we added in $H$-band data: 621 out of 665 galaxies (i.e., 93\%) had an $H$-band magnitude.

\subsection{The FUV-Selected Sample}

The selection of the FUV sources was carried out over the same area of sky as for the FIR-selected sample (that is, excluding areas contaminated by cirrus). We have checked that it is equivalent to selecting lines of sight with $E(B-V)<0.08 \mathrm{mag}$ (Schlegel et al. 1998). Our aim was to build a galaxy sample as complete as possible down to FUV $=17 \mathrm{mag}$. Galaxies are often resolved in the FUV and, as such, can be shredded into multiple fragments by the standard GALEX pipeline (e.g., Buat et al. 2005; Seibert et al. 2005), which is optimized to find and extract point sources. As a consequence we decided to preselect all the FUV sources brighter than FUV(pipeline) $=17.5 \mathrm{mag}$ (as estimated by the GALEX pipeline), where the FUV magnitudes were corrected for the Galactic extinction before selecting at FUV $=17.5$ mag. The star-galaxy separation was made by cross-correlating the sample with the HyperLeda and 2MASS databases. As for the FIR-selected sample, we performed all the FUV photometry manually. We estimated the level of completeness due to the shredding. Indeed, only sources brighter than FUV(pipeline) $=17.5 \mathrm{mag}$ were preselected because certain galaxies might, in their totality, be brighter than this limit, but they were cataloged with a lower flux because fainter subparts were detected, extracted, and mea-

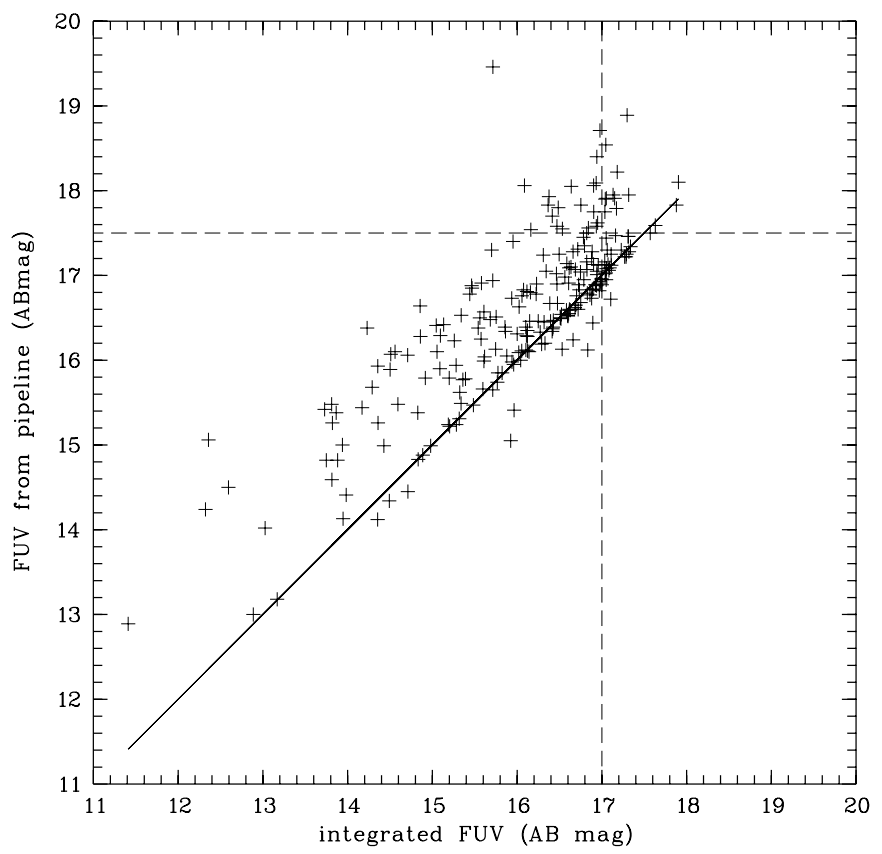

FIG. 1.-FUV magnitude from this paper ( $x$-axis) against the FUV magnitude from the pipeline (MAST archive) for the FIR-selected galaxies brighter than FUV $=18 \mathrm{mag}$. The dashed lines represent the limits applied for the FUV selection (see text for details).

sured individually by the pipeline. We used the FIR-selected sample to quantify this effect. We selected 238 galaxies in this sample brighter than FUV(total) $=18$ mag, where "total" means the fully integrated magnitude. For these galaxies we compared the integrated FUV magnitude that we measured manually with the FUV magnitude given by the pipeline as posted in the MAST archives. The result of the comparison is plotted in Figure 1. As expected, the pipeline underestimates the FUV flux, sometimes by a very large factor. Our initial selection of the FUV sources at FUV(pipeline) $=17.5 \mathrm{mag}$ (Fig. 1, horizontal dashed line) ensures us a completeness that is larger than $95 \%$ for galaxies brighter than FUV(total) $=16 \mathrm{mag}$ and of $80 \%$ for galaxies with $16 \mathrm{mag}<\mathrm{FUV}<17 \mathrm{mag}$. Therefore, in the following we adopt a FUV(total) $=17 \mathrm{mag}$ (Fig. 1, vertical dashed line $)$ as the lower flux limit to our sample; 762 galaxies have FUV $<17$ mag.

The FIR fluxes of these galaxies were mainly taken from the IRAS Faint Source Catalog (FSC; Moshir et al. 1992), and if an FSC flux was not available, it was measured by us using the Scan Processing and Integration Facility. This sample was cleaned in the same way as the FIR-selected sample: we accounted for confusion effects within the IRAS beam by again searching for neighbors in the 2MASS and NVSS catalogs. Dubious cases (several candidates) were checked individually. Eighteen galaxies were not detected by $I R A S$ at all, 123 were not detected at $60 \mu \mathrm{m}$ and an upper limit of 0.2 Jy was adopted (as given in the IRAS Faint Source Catalog), and 39 objects were affected by confusion. In the end we compiled a sample of 705 galaxies without confusion and for which detections or upper limits at $60 \mu \mathrm{m}$ were available. Radial velocities and morphological types were added from HyperLeda and NED. All sources had a radial velocity measurement; 51 galaxies had no morphological type. As for the FIR-selected sample, we only retained the 656 galaxies with $v>$ $1000 \mathrm{~km} \mathrm{~s}^{-1}$. Once again, 38 galaxies have been mentioned to have an active nucleus, and 44 have been classified E-S0. Sixhundred-six of the 656 galaxies have been observed by IRAS without any confusion, and 533 have been detected at $60 \mu \mathrm{m}$. Of the 
TABLE 2

FUV-Selected Sample

\begin{tabular}{|c|c|c|c|c|c|c|}
\hline Galaxy Name & $\begin{array}{l}\text { FUV } \\
\text { (mag) }\end{array}$ & $\begin{array}{l}F_{60} \\
(\mathrm{Jy})\end{array}$ & $\begin{array}{l}F_{100} \\
(\mathrm{Jy})\end{array}$ & $\begin{array}{c}c z \\
\left(\mathrm{~km} \mathrm{~s}^{-1}\right)\end{array}$ & $\begin{array}{c}H \\
(\mathrm{mag})\end{array}$ & Type \\
\hline PGC 158 & $16.8^{\circ}$ & 1.65 & 3.15 & 19199 & 12.02 & I \\
\hline PGC 229 . & 16.82 & 0.65 & 2.10 & 6202 & 12.41 & $\mathrm{SABC}$ \\
\hline PGC $282 \ldots \ldots \ldots \ldots \ldots$ & 16.43 & 0.28 & 0.66 & 11406 & 12.76 & $\mathrm{Sa}$ \\
\hline PGC 305. & 15.49 & . & .. & 3112 & 12.75 & $\mathrm{Sc}$ \\
\hline PGC $312 \ldots \ldots \ldots \ldots \ldots$ & 15.50 & 1.04 & 3.27 & 3816 & 10.53 & $\mathrm{SBb}$ \\
\hline
\end{tabular}

Notes.-Col. (1): PGC (from HyperLeda) or 2MASS number (only three objects have only a 2MASS number; they are at the end of the table). Col. (2): FUV magnitude (AB scale) corrected for Galactic extinction (see text). Col. (3): Flux at $60 \mu \mathrm{m}$. When no value is quoted an upper limit of $0.2 \mathrm{Jy}$ is adopted. Col. (4): Flux at $100 \mu \mathrm{m}$. When no value is quoted a mean ratio for $F_{60} / F_{100}$ is adopted (see text). Col. (5): Radial velocity from HyperLeda or NED by order of preference (the values taken from NED are indicated by an "N"). Col. (6): Total $H$ magnitude from 2MASS. Col. (7): Morphological type from HyperLeda or NED (the values taken from NED are preceded by a period). Table 2 is published in its entirety in the electronic edition of the Supplement. A portion is shown here for guidance regarding its form and content.

galaxies, $89 \%$ have an $H$ magnitude in $2 \mathrm{MASS}$. The sample of the 606 FUV-selected galaxies, without confusion and for which a detection or an upper limit at $60 \mu \mathrm{m}$ is available, is presented in Table 2. As for the FIR-selected sample, when the galaxies are not detected at $100 \mu \mathrm{m}$ we estimate the flux by using the mean value $F_{60} / F_{100}=0.4(\sigma=0.2)$ found for the galaxies in our sample detected at both wavelengths.

\section{THE LUMINOSITY AND REDSHIFT DISTRIBUTIONS OF THE SAMPLE GALAXIES}

The FIR- and FUV-selected samples are likely to be biased toward FIR- and FUV-strong emitters. As a consequence we expect the distribution of the FIR to FUV luminosities to be different within each sample, as has been found in previous studies (Buat et al. 2005; Martin et al. 2005; Iglesias-Páramo et al. 2006).

\subsection{Flux Distributions}

To highlight selection effects, in Figure 2 we plot $F_{60}$ versus $F_{\mathrm{FUV}}$ for the whole sample, splitting them according to $\left(L_{60}+L_{\mathrm{FUV}}\right)$ and radial velocity (i.e., their distances). The dotted diagonal lines are the loci of constant $F_{60} / F_{\mathrm{FUV}}$ and can thus be considered as lines of relatively constant dust attenuation (the derivation of a quantitative attenuation is in fact based on an analysis of the total infrared emission [TIR; e.g., Buat et al. 2005] and not solely on $60 \mu \mathrm{m}$ emission, but for the purpose of the present qualitative discussion we can safely neglect this difference). From Figure 2 it is obvious that the FIR selection focuses on extinguished galaxies, whereas the FUV selection is biased toward galaxies with a low $F_{60} / F_{\mathrm{FUV}}$ ratio. Indeed the FIR-selected sample exhibits a long tail toward large $F_{60} / F_{\mathrm{FUV}}$ ratios. More distant galaxies are selected in the FIR than in the FUV (e.g., Iglesias-Páramo et al. 2006; Xu et al. 2006; and the discussion below).

\subsection{Monovariate Luminosity Functions}

The very different distributions found in Figure 2 are the direct consequence of the shapes of the individual FIR and FUV
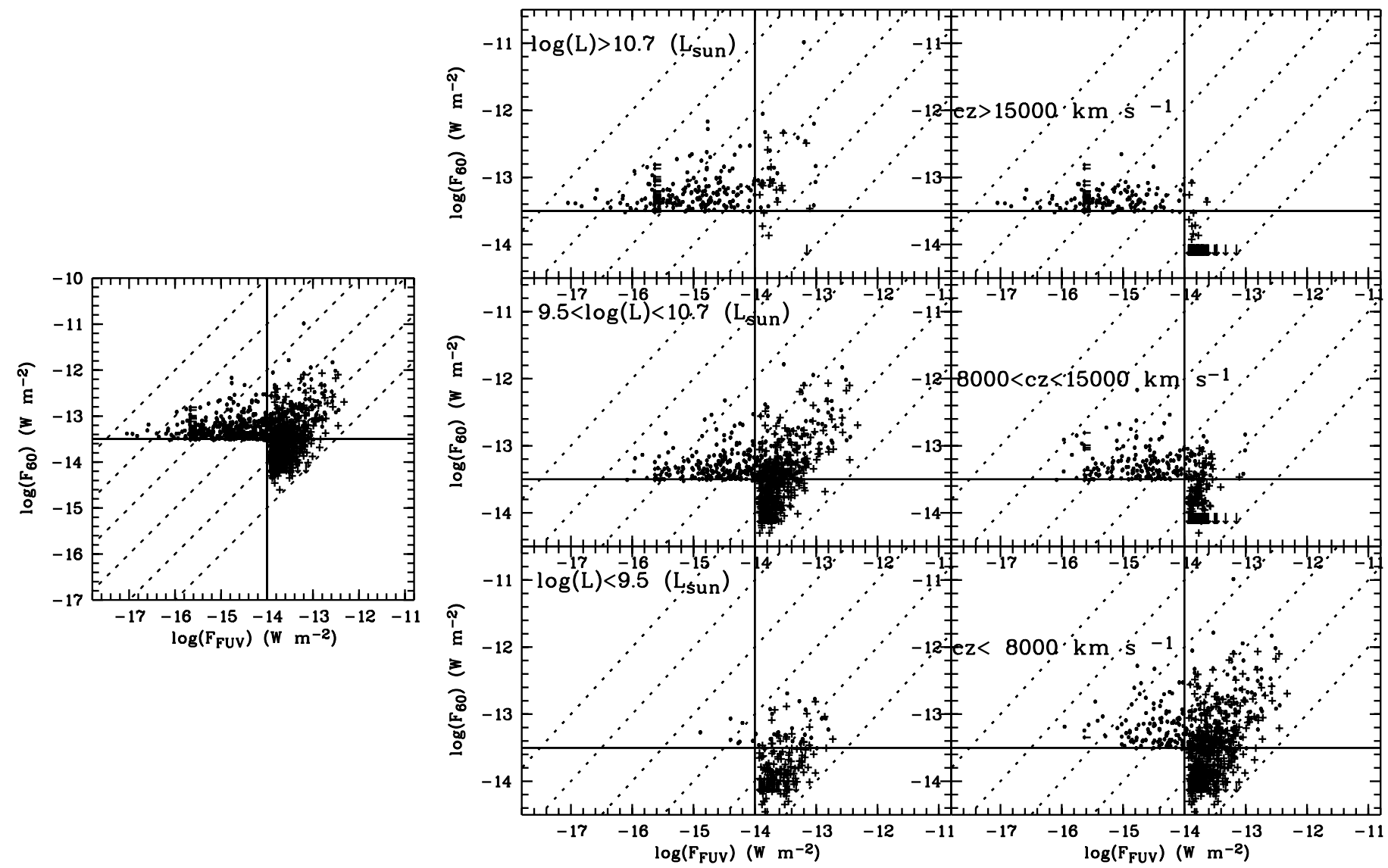

FIg. 2.-FUV flux against the FIR for the FIR- (circles) and FUV-selected (crosses) samples. Dotted lines, Constant FIR-to-FUV flux ratio; solid lines, FIR (horizontal line) and FUV (vertical line) selections. In the right panels the samples are split according to the luminosity ( $L_{60}+L_{\mathrm{FUV}}$ ) or distance (as traced by their velocity) of the galaxies. Arrows indicate upper limits. No upper limit is plotted in the selection per bin of luminosity since $L_{60}+L_{\mathrm{FUV}}$ is defined only for galaxies detected at both wavelengths. [See the electronic edition of the Supplement for a color version of this figure.] 
luminosity functions (LFs). These are known to be very different (e.g., Buat \& Burgarella 1998; Takeuchi et al. 2005a; IglesiasPáramo et al. 2006). We have calculated the FUV and $60 \mu \mathrm{m}$ luminosity functions for our FUV- and FIR-selected samples, respectively, using the $1 / V_{\max }$ and the $C^{-}$methods and the recipes of Takeuchi et al. (2000). We excluded galaxies known to be active since we are only interested in a measurement of the star formation (non-AGN) activity from the FIR and the FUV emissions. The optimal bin size was determined systematically by the formula of Takeuchi (2000), and the error bars were obtained by bootstrap resampling (for details, see Takeuchi et al. 2000). We checked that the $K$-corrections are negligible; the study is restricted to the very nearby universe (see Wyder et al. 2005). We also examined the completeness of the sample in an LF analysis, using the number counts of galaxies. Our sample again turned out to be highly complete, brighter than 17 mag for the UV and brighter than 0.6 Jy for the FIR sample. The LFs are reproduced in Figure 3 and compared to the local LFs built by Wyder et al. (2005) in FUV and Takeuchi et al. (2003) at $60 \mu \mathrm{m}$. The agreement is very good, thereby encouraging us to believe that our samples are representative of nearby universe populations. For both selections, the faintest bins appear to be underestimated. In the following we exclude these bins from the analysis. The very similar results found with both methods $\left(1 / V_{\max }\right.$ and the $C^{-}$) validate the use of $V_{\max }$ in the following analyses.

The extension of the $60 \mu \mathrm{m}$ LF toward bright galaxies, as compared to the FUV one, implies that we see intrinsically brighter and more distant galaxies in an FIR-selected sample.

\section{DO WE SEE THE SAME GALAXIES IN THE FIR AND FUV?}

One of the fundamental questions to address is, do we see the same universe in the FIR and FUV, or do we miss galaxy populations when working at a single wavelength? For example, Adelberger \& Steidel (2000) claimed to see all the star formation at $z=3$ with a purely UV selection criterion. At low redshift, $\mathrm{Xu}$ et al. (2006) concluded that we globally see the same galaxies, but the statistics were poor, and subtle effects might have been difficult to examine. We will now re-examine these questions with our much larger samples. Given the very different shapes of the luminosity functions and the different volumes sampled by each selection (FIR and FUV), the comparison is not trivial (e.g., $\mathrm{Xu}$ et al. 2006). We must also define intrinsic properties independent of the wavelength selections to compare the samples. Since we are interested in the measure of the star formation, we focus on the distribution of bolometric luminosities from young stars.

\subsection{Bolometric Luminosity Functions from Young Stars}

A very crude and spread-out way of estimating the total energy coming from newly formed stars is simply to add $L_{\mathrm{FUV}}$ and $L_{60}$ (e.g., Martin et al. 2005; Wang \& Heckman 1996; Xu et al. 2006). We can also make a more sophisticated analysis by first calculating the total IR luminosity $\left(L_{\mathrm{TIR}}\right)$ to account for all of the emission from dust. In addition to $L_{\mathrm{TIR}}$ having more physical significance than $L_{60}$, post-IRAS infrared observations (the Infrared Space Observatory, Spitzer, and Astro-F/AKARI) have been made at different wavelengths from IRAS, and there again the comparison is usually made through the TIR emission. Therefore, $L_{\mathrm{TIR}}$ is better suited than $L_{60}$ for a comparison with studies at higher redshift. We calculate $L_{\mathrm{TIR}}$ by combining the fluxes at 60 and $100 \mu \mathrm{m}$ according to the recipes in Dale et al. (2001).

Calculating $L_{\mathrm{FUV}}$ as $\nu L_{\nu}$ is a good measure of the total luminosity between 1200 and $3600 \AA$ as long as no dust attenuation occurs: using Starburst 99 under the hypothesis of a constant star
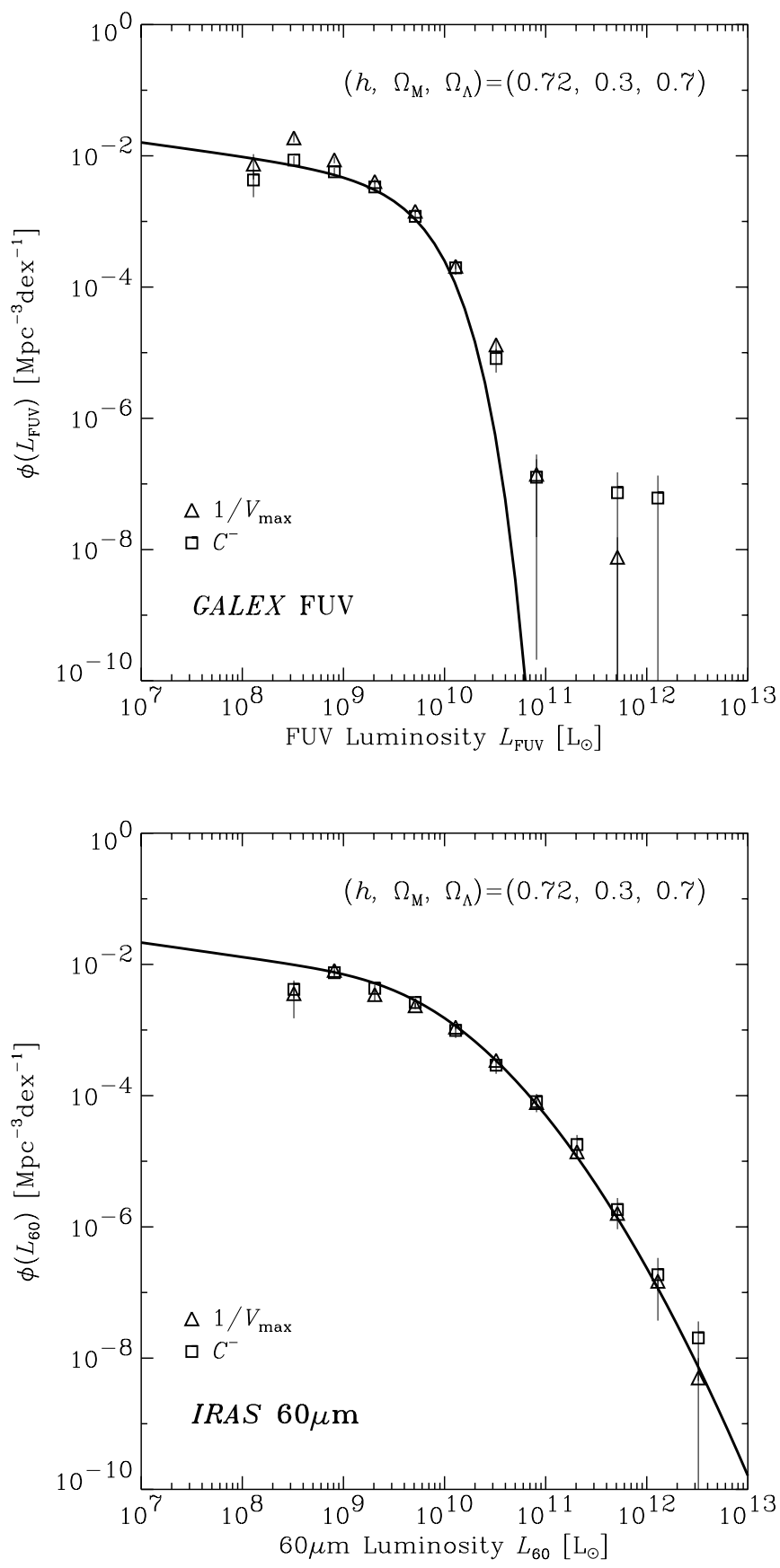

FIG. 3.-FUV and $60 \mu \mathrm{m}$ LFs for the FUV- and FIR-selected samples. The LFs are estimated by the $1 / V_{\max }$ method (triangles) and the $C^{-}$method (squares). We also overplot the analytic FUV LF from Wyder et al. (2005) and the $60 \mu \mathrm{m} \mathrm{LF}$ of Takeuchi et al. (2003) (solid lines). Errors $(1 \sigma)$ are calculated by bootstrap resampling. [See the electronic edition of the Supplement for a color version of this figure.]

formation rate over 100 Myr leads to $L(1200-3600 \AA) \sim 0.8 L_{\mathrm{FUV}}$. Nevertheless, a dust attenuation which is strongly dependent on the wavelength may induce large variations in this relation: as an example, using Calzetti et al.'s (2000) attenuation law and a color excess $E(B-V)=0.3$ [corresponding to $A(\mathrm{FUV})=3 \mathrm{mag}$ and a TIR-to-FUV flux ratio of $\sim 30]$ implies $L(1200-3600 \AA) \sim$ $1.3 L_{\mathrm{FUV}}$. Hirashita et al. (2003) showed that the effect on the total estimate of the star formation rate is not large as long as the contribution of the TIR and FUV emissions are added (the uncertainties are reduced by the large contribution of the TIR emission to the total bolometric emission). Following Hirashita et al. (2003), 


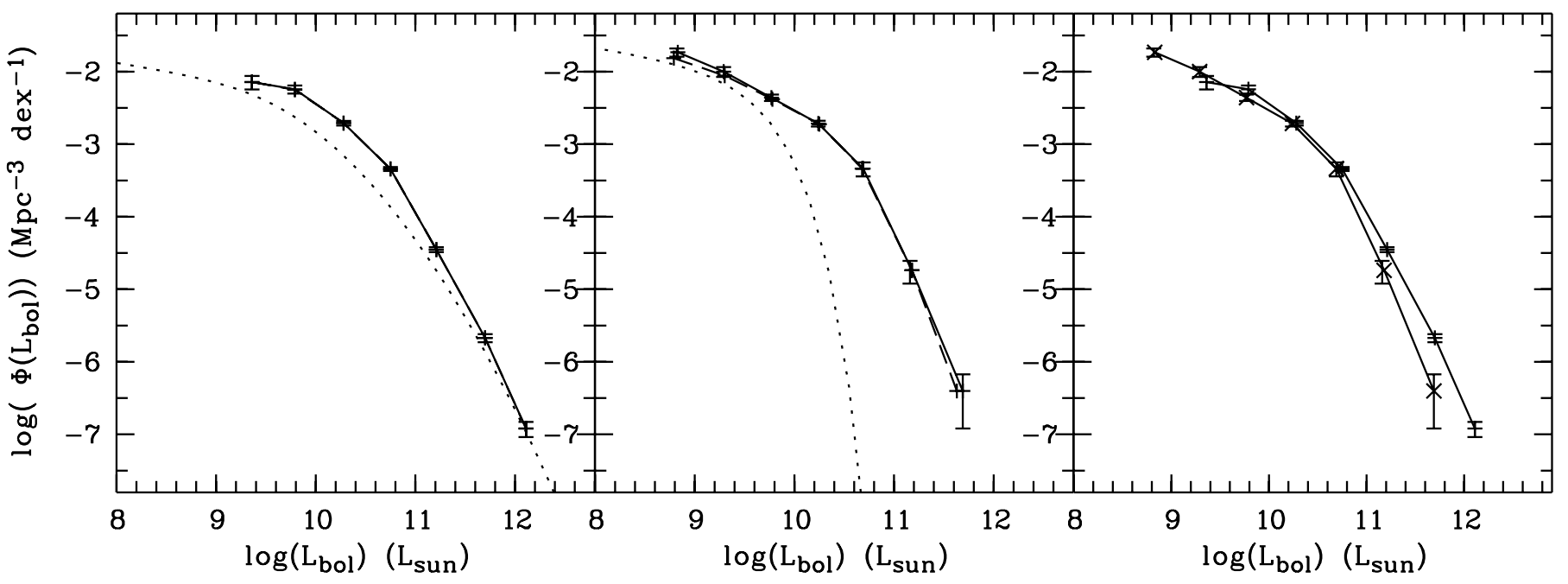

FIG. 4.- Bolometric LFs calculated for the two considered scenarios (scenario 1 , solid line; scenario 2 , dashed line). The quantity $L_{\mathrm{bol}}$ is defined as $L_{\mathrm{bol}}=L_{\mathrm{FuV}}+$ $(1-\eta) L_{\mathrm{TIR}}$, and the $1 \sigma$ error bars are overplotted. Left: FIR-selected sample. The monochromatic $(60 \mu \mathrm{m}) \mathrm{LF}$ from Takeuchi et al. (2005b) is represented as a dotted line. Middle: FUV-selected sample. The monochromatic (FUV) LF (Wyder et al. 2005) is represented as a dotted line. Right: Comparison of the bolometric LF for the FUV-selected and FIR-selected samples. Crosses and the lower line are used for the FUV selection, and plus signs and the upper line for the FIR selection. Only scenario 1 is considered. [See the electronic edition of the Supplement for a color version of this figure.]

we also account for the dust heating by stars older than $100 \mathrm{Myr}$, which generates a TIR emission not related to the recent star formation; the total luminosity of young stars is thus expressed as $L_{\mathrm{bol}}=L_{\mathrm{FUV}}+(1-\eta) L_{\mathrm{TIR}}$, where $\eta$ is the fraction of the TIR emission not related to the star formation. The value of $\eta$ is found to be between 0.2 and 0.4 in the nearby universe (Bell 2003; Hirashita et al. 2003; Iglesias-Páramo et al. 2006). Hereafter we adopt $\eta=0.3$. The total luminosity of young stars can therefore be written as $L_{\mathrm{bol}}=L_{\mathrm{FUV}}+0.7 L_{\mathrm{TIR}}$. This formula is established for local star-forming galaxies and confirmed to be valid for nearby galaxies selected in the FIR or FUV (Iglesias-Páramo et al. 2004, 2006). Nevertheless, in galaxies forming stars very actively, the entire TIR luminosity is likely to be related to the recent star formation in starburst galaxies (e.g., Hirashita et al. 2003).

The bolometric luminosity function from young stars is calculated within each sample using the $1 / V_{\max }$ weighting method. We test the influence of $K$-corrections by interpolating the FUV and NUV magnitudes according to the redshift, and they are found to be negligible in both samples. As for the derivation of the monovariate luminosity functions $(\S 3.2)$, galaxies known to be active are dropped. We consider the entire sample, including upper limits. Therefore, the $V_{\max }$ value of each galaxy is defined by its luminosity and the detection limit relevant for the selection (FUV or FIR), since the volume sampled by the other wavelength is infinite as soon as upper limits are included. The adopted limits are that of the IRAS Point Source Catalog for the FIR-selected sample $(0.6 \mathrm{Jy}$ at $60 \mu \mathrm{m})$ and FUV $=17 \mathrm{mag}$ for the FUV-selected sample (by construction). Taking into account the upper limits, we consider two extreme scenarios in calculating the luminosity functions: in scenario 1, upper limits are considered as true detections, and in scenario 2 , a flux equal to zero is adopted for a nondetection.

In Figure 4 we plot the bolometric LF $\phi\left(L_{\mathrm{bol}}\right)$ for each sample as compared to the monochromatic ones at $60 \mu \mathrm{m}$ (Takeuchi et al. 2005b) and at $1530 \AA$ (Wyder et al. 2005). The two scenarios adopted to calculate the bolometric LF agree very well for the FIR-selected sample. It therefore appears that the $60 \mu \mathrm{m}$ luminosity is a robust tracer of the luminosity of young stars: the bolometric LF appears to be shifted as compared to the LF at $60 \mu \mathrm{m}$ by at most a factor of $\sim 3$ (for intermediate luminosities). The dif- ference between LFs decreases as the luminosity increases: for the highest luminosities the $60 \mu \mathrm{m}$ luminosity function is similar to the bolometric one.

The two scenarios adopted to calculate the bolometric LF in the FUV-selected sample also agree quite well: slight differences (lower than 0.2 dex in the vertical direction and 0.1 dex in the horizontal) are visible at the faint and luminous ends, but they are smaller than the $1 \sigma$ errors calculated for each scenario.

Even within a FUV selection the FUV flux alone (without any correction) misses a large part of the total emission of FUVselected galaxies. Whereas the FUV flux appears to be a reliable estimator of the bolometric emission from young stars in lowluminosity galaxies $\left(L_{\text {bol }}<2.5 \times 10^{9} L_{\odot}\right)$, the difference increases very fast with luminosity: the FUV luminosity is $\sim 5$ times lower than the bolometric one for $L_{\mathrm{bol}} \sim 10^{10} L_{\odot}$, and the discrepancy reaches a factor of $\sim 500$ for $L_{\mathrm{bol}}=3 \times 10^{10} L_{\odot}$. This trend has to be related to the relation found between the luminosities (or star formation rates) of galaxies and their dust attenuation (Wang \& Heckman 1996; Buat \& Burgarella 1998; Hopkins et al. 2001; Sullivan et al. 2001; and the discussion in $\S 4.3$ ). As a consequence, large luminosity-dependent corrections must be applied to the FUV emission in order to retrieve all of the bolometric luminosity of young stars (i.e., the recent star formation rate) of FUV-selected galaxies. In the following, and in order to simplify the discussions, we adopt scenario 1 for the calculations; that is, we include upper limits as detections.

Figure 4 compares the bolometric LF for both samples. It can be seen that both luminosity functions are consistent for intermediate luminosities: in the nearby universe these galaxies are detected equally well in FIR and FUV. For bolometric luminosities larger than $5 \times 10^{10} L_{\odot}$, the bolometric LF derived from the FIR selection is higher than that derived from the FUV, and the discrepancy increases with the luminosity: we miss intrinsically bright galaxies which appear much fainter in the FUV (see $\S 4.3)$. The shallowness of the IRAS survey does not allow us to compare the distributions at low luminosities $\left(L_{\text {bol }} \lesssim 2 \times 10^{9} L_{\odot}\right)$.

\subsection{Energy Distributions}

In order to estimate what fraction of the energy emitted by young stars in the nearby universe is recovered from an FUV selection 


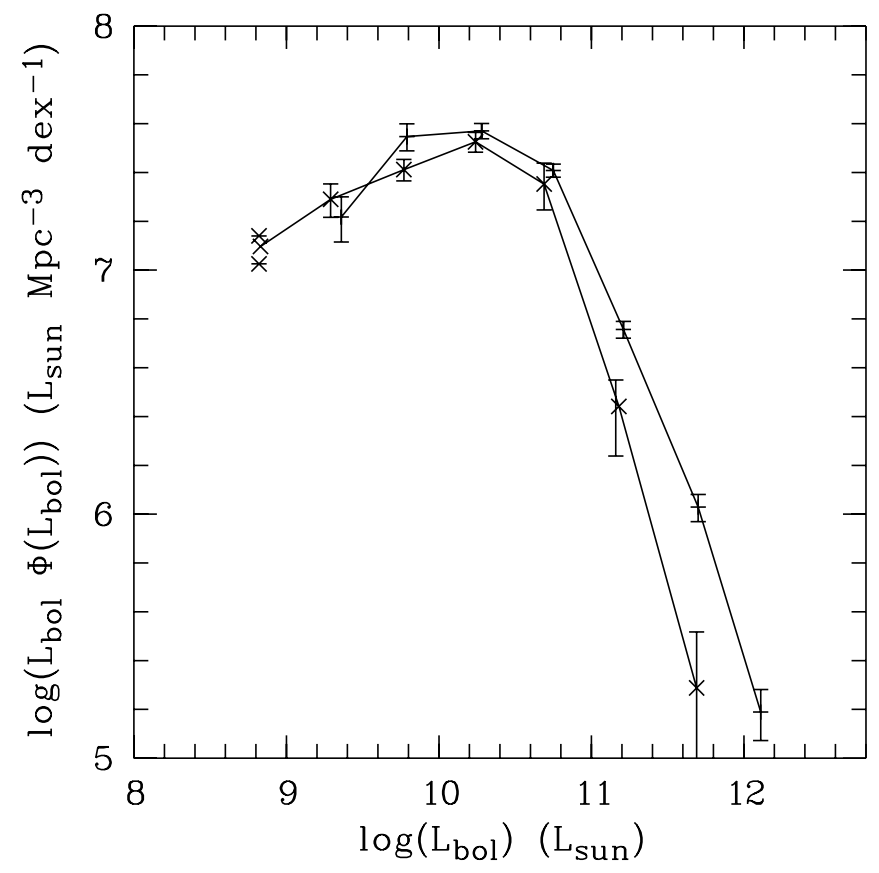

FIG. 5.-Energy distribution $\left(\log \left[L_{\mathrm{bol}} \phi\left(L_{\mathrm{bol}}\right)\right]\right)$ as a function of $\log \left(L_{\mathrm{bol}}\right)$ for the FUV- and FIR-selected samples. Same symbols as in Fig. 4. The $1 \sigma$ error bars are also overplotted. [See the electronic edition of the Supplement for a color version of this figure.]

and how much from an FIR selection, we have calculated the $L_{\text {bol }} \phi\left(L_{\text {bol }}\right)$ product, which represents the energy contribution of galaxies with a given $L_{\text {bol }}$ luminosity to the luminosity density in the local universe as a whole. The distributions are shown in Figure 5 (all the calculations are made with scenario 1, i.e., with upper limits considered as detections). The distributions are marginally consistent at the $1 \sigma$ level at intermediate luminosity. As for the luminosity functions ( $\S 4.1)$, a discrepancy appears at high luminosity and increases with luminosity: at $L_{\text {bol }} \sim 5 \times$ $10^{10} L_{\odot}$ the FUV selection systematically underestimates the luminosity density by a factor of $\sim 1.5$, and the factor reaches $\sim 5$ for $L_{\mathrm{bol}} \sim 3 \times 10^{11} L_{\odot}$. At the faint end it seems that the situation is reversed, with a large number of low-luminosity UV-selected galaxies not being present in our FIR selection. Deeper FIR ob- servations will be necessary to confirm or negate this trend: future $A K A R I$ observations will allow us to address this issue.

\section{3. $L_{\mathrm{TIR}} / L_{\mathrm{FUV}}$ Distributions}

Analyzing the $L_{\mathrm{TIR}} / L_{\mathrm{FUV}}$ ratio is another way to compare the FIR and FUV selection effects (Martin et al. 2005; Xu et al. 2006). Indeed, the $L_{\mathrm{TIR}} / L_{\mathrm{FUV}}$ ratio has a physical significance, since it is directly related to the dust attenuation in star-forming galaxies (e.g., Buat \& Xu 1996; Gordon et al. 2000; Buat et al. 2005). This ratio gives us information about the dust obscuration, as well as about the differences and/or similarities in the galaxies selected in different ways (FIR vs. FUV). Hereafter we deal with the $L_{\mathrm{TIR}} / L_{\mathrm{FUV}}$ ratio, taking in mind that it can be calibrated in absolute dust attenuation at FUV wavelengths using, for example, the formulae of Buat et al. (2005):

$$
\begin{aligned}
A(\mathrm{FUV})[\mathrm{mag}]= & -0.0333\left(\log \frac{L_{\mathrm{TIR}}}{L_{\mathrm{FUV}}}\right)^{3}+0.3522\left(\log \frac{L_{\mathrm{TIR}}}{L_{\mathrm{FUV}}}\right)^{2} \\
& +1.1960\left(\log \frac{L_{\mathrm{TIR}}}{L_{\mathrm{FUV}}}\right)+0.4967
\end{aligned}
$$

Figure 6 shows the variation of $L_{\mathrm{TIR}} / L_{\mathrm{FUV}}$ as a function of $L_{\mathrm{bol}}$ for the two samples under consideration (FIR-and FUV-selected). It is also useful to consider the variation of this ratio as a function of the "monochromatic" luminosities (at $60 \mu \mathrm{m}$ or in the FUV band alone). These plots are found in Figure $6 ; L_{\mathrm{TIR}} / L_{\mathrm{FUV}}$ (i.e., the dust attenuation) is found to increase with $L_{\mathrm{TIR}}$ and with $L_{\mathrm{bol}}$ in both samples. Such an increase of $L_{\mathrm{TIR}} / L_{\mathrm{FUV}}$ with the TIR luminosity confirms previous results (Wang \& Heckman 1996; Buat \& Burgarella 1998; Hopkins et al. 2001; Sullivan et al. 2001) and appears to be robust against selection effects. The similarity between the trends found with $L_{\mathrm{TIR}}$ and $L_{\mathrm{bol}}$ is obvious and is due to the dominant contribution of the TIR luminosity to the bolometric luminosity, as compared to the FUV contribution (see Fig. 4). The trend is steeper and more scattered for the FIR selection than for the FUV. Very different trends are found within each sample with $L_{\mathrm{FUV}}$ : a strong decrease of $L_{\mathrm{TIR}} / L_{\mathrm{FUV}}$ with $L_{\mathrm{FUV}}$ is observed for the FIR-selected sample, whereas a very loose positive correlation is found for the FUV selection (correlation coefficient equal to 0.2 ), making irrelevant any correction of the dust attenuation based on the observed FUV luminosity alone. It confirms the

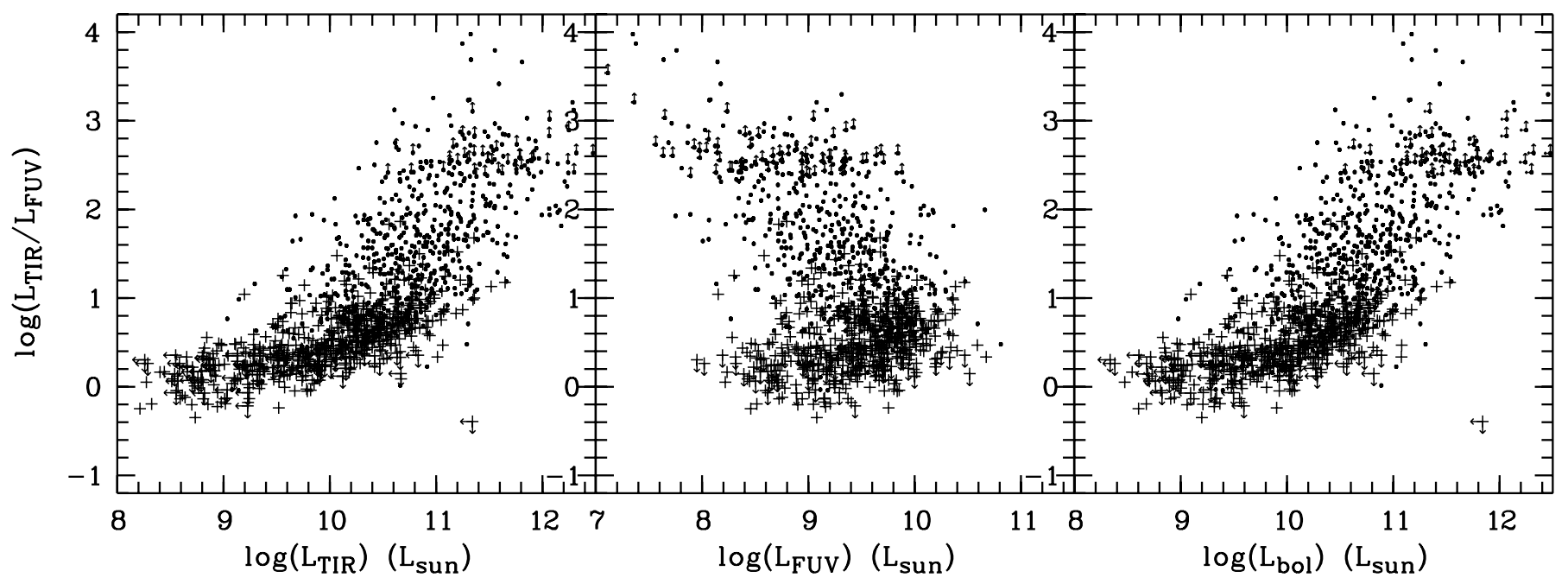

FIG. 6. $-L_{\mathrm{TIR}} / L_{\mathrm{FUV}}$ ratio vs. $L_{\mathrm{TIR}}, L_{\mathrm{FUV}}$, and $L_{\mathrm{bol}}$ for the FIR-selected sample (circles) and FUV-selected sample (crosses). [See the electronic edition of the Supplement for a color version of this figure.] 
results of Iglesias-Páramo et al. (2006) that the FUV emission emerging from galaxies selected in the FIR represents only a very small fraction of the total luminosity emitted by the young stars. It is worth noting that galaxies selected with a very high FUV luminosity $\left(\gtrsim 2 \times 10^{10} L_{\odot}\right)$ exhibit a rather moderate $L_{\mathrm{TIR}} / L_{\mathrm{FUV}}$ ratio [i.e., a small attenuation: $A(\mathrm{FUV})=1.5 \pm 0.5 \mathrm{mag}$ using eq. (1)], some of them having a dust attenuation as low as $\sim 0.5 \mathrm{mag}$, corresponding to $L_{\mathrm{TIR}} / L_{\mathrm{FUV}} \sim 1$. These galaxies are called $\mathrm{UV}$ luminous galaxies by Heckman et al. (2005). A subclass of these galaxies is thought to contain the analogs of the more distant Lymanbreak galaxies (Hoopes et al. 2006 and the discussion below). As noted in the previous section, we must also account for the different volumes explored within each selection in order to secure the trends. To this end we now calculate the weighted distributions of $L_{\mathrm{TIR}} / L_{\mathrm{FUV}}$ as a function of $L_{\mathrm{bol}}$. We divide the sample into bins of $L_{\text {bol }}$ (bin size $=0.5 \mathrm{dex}$ ), and for each bin we calculate the averaged ratio $R=\log \left(L_{\mathrm{TIR}} / L_{\mathrm{FUV}}\right)$ and its standard deviation as follows:

$$
\begin{gathered}
\left\langle R\left(L_{\mathrm{bol}}\right)\right\rangle=\frac{\sum_{i} \omega_{i} R_{i}}{\sum_{i} \omega_{i}}, \\
\sigma^{2}\left(L_{\mathrm{bol}}\right)=\frac{\sum_{i} \omega_{i}\left[R_{i}-\left\langle R\left(L_{\mathrm{bol}}\right)\right\rangle\right]^{2}}{\sum_{i} \omega_{i}},
\end{gathered}
$$

where $\omega_{i}$ is the weight for the $i$ th galaxy, practically $1 / V_{\max }$, and $V_{\max }$ is calculated as for the bolometric luminosity functions under scenario 1 (with upper limits treated as true detections). Indeed, scenario 2 , which essentially defines the nondetected sources as having zero flux, is irrelevant to the analysis of the $L_{\mathrm{TIR}} / L_{\mathrm{FUV}}$ ratio. Adopting scenario 1 is a conservative approach when searching for differences between the FIR- and FUV-selected samples: the nondetections lead to upper limits for $L_{\mathrm{TIR}} / L_{\mathrm{FUV}}$ in the FUVselected sample and lower limits in the FIR-selected sample. The results of the calculations are plotted in Figure 7. Both samples give similar trends at low and intermediate luminosities, but the volume corrections cannot completely compensate for the very different distributions seen in Figure 6 for the high luminosities. Whereas the $L_{\mathrm{TIR}} / L_{\mathrm{FUV}}$ ratio continues to increase with luminosity for FIR-selected galaxies, it shows a clear flattening for FUVselected galaxies brighter than $5 \times 10^{10} L_{\odot}$, and the $L_{\mathrm{TIR}} / L_{\mathrm{FUV}}$ ratio seems to reach an asymptotic value which corresponds to a dust attenuation $A(\mathrm{FUV}) \simeq 2.5 \mathrm{mag}$.

For the nearby universe, Bell (2003) analyzed a sample of nearby galaxies and found that $L_{\mathrm{TIR}} / L_{\mathrm{FUV}} \simeq\left(L_{\mathrm{TIR}} / 10^{9}\right)^{0.5}$, where $L_{\mathrm{TIR}}$ is expressed in solar units. The galaxies selected by Bell have $L_{\mathrm{TIR}}<10^{11} L_{\odot}$. His mean relation (transformed to the quantities used here: $L_{\mathrm{TIR}} / L_{\mathrm{FUV}}$ and $\left.L_{\mathrm{bol}}\right)$ is shown in Figure 7. The general behavior is similar to that found for our FUV selection, where the Bell relation gives lower a $L_{\mathrm{TIR}} / L_{\mathrm{FUV}}$ ratio for a given luminosity but still within our $1 \sigma$ error bars. We can also compare our results to those obtained from a GALEX-IRAS comparison (Martin et al. 2005; Xu et al. 2006). To perform this comparison we have transformed the $60 \mu \mathrm{m}$ luminosity used in those works into a TIR luminosity by applying a factor of 2.5 (Takeuchi et al. 2005b, 2006). Xu et al. (2006) did not find a significant difference between the FIR and the FUV selection. The mean relation they found is overplotted in Figure 7. This is consistent with the present analysis, especially for intermediate luminosities. The consistency is only marginal at low luminosity $\left(L_{\mathrm{bol}} \leqslant 5 \times 10^{9} L_{\odot}\right)$ and for the last bin in our FUV-selected sample with $L_{\mathrm{bol}}>10^{11} L_{\odot}$; we obtain larger values of $L_{\mathrm{TIR}} / L_{\mathrm{FUV}}$ than Xu et al. These differences can be explained if we go back to the sample selections. The samples used by Xu et al. were smaller and shallower: the FUV-selected

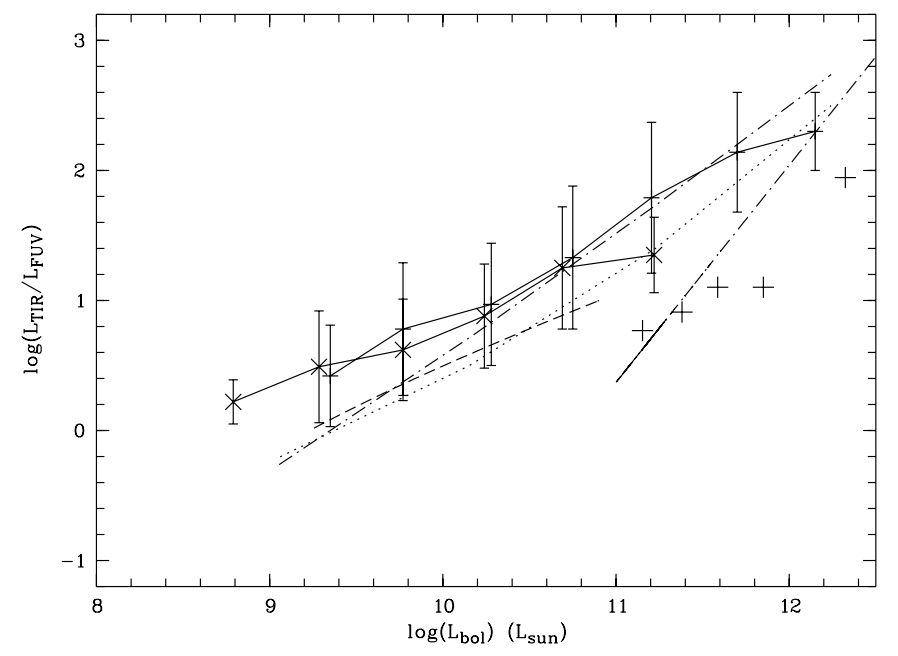

FIG. 7.-Mean $L_{\mathrm{TIR}} / L_{\mathrm{FUV}}$ ratio vs. $L_{\mathrm{bol}}$ calculated for the FUV-selected sample (lower solid line and crosses) and the FIR-selected sample (upper solid line and plus symbols). The errors $(1 \sigma)$ are overplotted as vertical bars. The dotted line is from Martin et al. (2005), the upper dot-dashed line is from $\mathrm{Xu}$ et al. (2006), the black dashed line is from Bell (2003), the lower dot-dashed line is from Reddy et al. (2006) (optically selected galaxies at $z \sim 2$ also observed at $24 \mu \mathrm{m}$ ), and the plus symbols at the right of the figure correspond to mean values per bin of luminosity for luminous blue galaxies at $z \sim 1$ from Burgarella et al. (2006). [See the electronic edition of the Supplement for a color version of this figure.]

sample contained only 94 objects brighter than NUV $=16$ mag, and the FIR-selected sample had only 161 galaxies (with a similar selection as in the present study). As a consequence, the high- and low-luminosity ranges are likely to be undersampled in the Xu et al. study. Another difference comes from the treatment of confused objects (i.e., galaxies not resolved by IRAS; see $\S 2$ ): $\mathrm{Xu}$ et al. included them in their analysis, whereas they are excluded from the present work. A reliable study of the difference of behavior between isolated and close pairs/interacting galaxies will be addressed with future $A K A R I$ data, whose spatial resolution will be much better than that of IRAS. Martin et al. (2005) used a combined sample (galaxies selected in FUV or at $60 \mu \mathrm{m}$ ) and analyzed the $L_{60} / L_{\mathrm{FuV}}$ distribution. Their mean relation (translated in $L_{\mathrm{TIR}} / L_{\mathrm{FUV}}$ and $L_{\mathrm{bol}}$ according to the definitions we adopt in this paper) is also overplotted in Figure 7. If we take into account the dispersions found in both studies (only ours are reported in the figure, but those of Martin et al. are similar), the results are consistent, although our mean values for $L_{\mathrm{TIR}} / L_{\mathrm{FUV}}$ are systematically higher than those obtained by Martin et al. At low luminosities, the relations begin to diverge. In this luminosity range the FUV selection is likely to dominate the Martin et al. sample, and their sample was built on an area 3 times smaller than ours, so we can expect some undersampling of these bins in their study.

It is also very interesting to use our results to search for a redshift evolution of the dust attenuation at a given bolometric luminosity. The comparison is not easy because only few high-redshift studies are based on accurate determinations of the rest-frame FUV and FIR luminosities. Nevertheless, with the advent of the Spitzer data the situation is evolving fast. Reddy et al. (2006) studied optically selected $z \sim 2$ galaxies which were also observed by Spitzer at $24 \mu \mathrm{m}$. Although the extrapolation from the observed MIR range to the total IR is not straightforward, they compare the $L_{\mathrm{TIR}} / L_{\mathrm{FUV}}$ ratio (their FUV at $1600 \AA$ is very similar to our FUV band) with $L_{\mathrm{TIR}}+L_{\mathrm{FUV}}$. The best fit they obtain is reproduced in Figure 7 (for $L_{\mathrm{bol}}>10^{11} L_{\odot}$, since they have access to only these galaxies). For $10^{11} L_{\odot}<L_{\text {bol }}<2.5 \times 10^{11} L_{\odot}$ the dust attenuation seems to be lower at $z=2$ than at $z=0$ as 
claimed by Reddy et al. But for intrinsically brighter objects, the $L_{\mathrm{TIR}} / L_{\mathrm{FUV}}$ ratios found by Reddy et al. are consistent with what we find in our FIR- and FUV-selected samples without invoking any evolution of the dust attenuation. Burgarella et al. (2006) selected Lyman-break galaxies at $z \sim 1$ in the Chandra Deep Field-South using GALEX data. One-fourth of these galaxies have a Spitzer detection at $24 \mu \mathrm{m}$. The mean values of $L_{\mathrm{TIR}} / L_{\mathrm{FUV}}$ obtained for these galaxies per bin of bolometric luminosity (bin size $=0.5 \mathrm{dex}$ ) are shown in Figure 7 . For these galaxies the dust attenuation (estimated through their $L_{\mathrm{TIR}} / L_{\mathrm{FUV}}$ ratio) is found to be consistent with that found by Reddy et al. for the same range of bolometric luminosities. A more complete comparison of the samples is forthcoming (Burgarella et al. 2008).

Analyses of both the bolometric luminosity functions and the $L_{\mathrm{TIR}} / L_{\mathrm{FUV}}$ ratio lead to the conclusion that a FUV selection misses some of the most heavily obscured and intrinsically brightest galaxies. Conversely, an FIR selection probably underestimates the contribution of intrinsically faint (in a bolometric sense) galaxies. Deeper FIR surveys are needed to confirm this effect. Our analysis is performed on galaxy samples excluding active and confused sources. Although only a few sources were excluded, the contribution of interacting systems and close pairs must be investigated with future $A K A R I$ data.

At higher redshift the general trend toward high-luminosity systems, together with the increase of $L_{\mathrm{TIR}} / L_{\mathrm{FUV}}$ with the luminosity, may argue for a gradual increase with redshift of the loss of star formation in FUV surveys. Nevertheless, this effect may be compensated for, at least in part, if the dust attenuation of UV/ optical-selected galaxies substantially decreases at high $z$, as suggested by Reddy et al. (2006). Statistical studies of FIR- and FUV-selected samples have to be performed at higher $z$ to investigate this issue. The SWIRE GALEX comparison performed by $\mathrm{Xu}$ et al. (2007) leads to no apparent difference between the $L_{\mathrm{TIR}} / L_{\mathrm{FUV}}$ ratios of star-forming galaxies between $z=0$ and 0.6 . An analysis of deep fields observed by GALEX and Spitzer MIPS is underway.

\section{SPECIFIC STAR FORMATION RATES}

Since we are dealing with star formation rates, galaxies classified as early type (E-S0) are excluded from the following analysis. Galaxies known to have an active nucleus are also excluded (as in $\S \S 3$ and 4). The present star formation efficiency of galaxies can be quantified by comparing their whole stellar mass to their present SFR. The specific SFR (SSFR) is defined as the ratio of the present SFR to the stellar mass: SSFR $=\mathrm{SFR} / M_{*}$. This SSFR is closely related to the so-called $b$-parameter, defined as the ratio of the present to past averaged SFR:

$$
b=\frac{\mathrm{SFR}}{\langle\mathrm{SFR}\rangle}=\frac{t(1-R) \mathrm{SFR}}{M_{*}},
$$

where $R$ is the fraction of recycled gas, usually taken to be between 0.3 and 0.5 , and $t$, the age of the galaxies, is assumed to be $\sim 13$ Gyr (e.g., Boselli et al. 2001). The total star formation rate is calculated by combining the TIR- and FUV-derived SFR, as done earlier by Hirashita et al. (2003) and more recently by IglesiasPáramo et al. (2006) for galaxies selected in a way very similar to in this work:

$$
\mathrm{SFR}_{\text {tot }}=\operatorname{SFR}\left(\mathrm{FUV}_{\mathrm{obs}}\right)+(1-\eta) \operatorname{SFR}(\mathrm{TIR}),
$$

with $\eta=0.3$. To undertake this analysis we need to estimate the total stellar mass in our galaxies. Most of them were detected by 2MASS; therefore, we decided to use their $H$-band luminosities

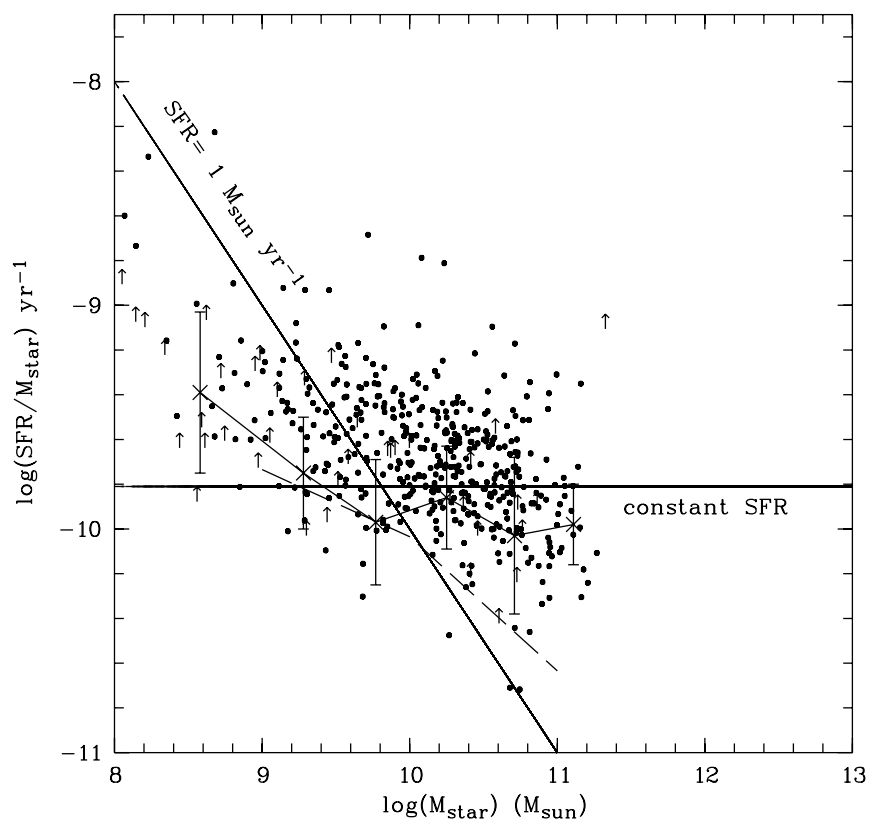

FIG. 8.-SSFR for the FUV-selected sample. The thin solid line shows the average SSFR, and $1 \sigma$ errors are overplotted. The horizontal line represents a constant SFR over the lifetime of the galaxy. The thick diagonal line corresponds to the present SFR equal to $1 M_{\odot} \mathrm{yr}^{-1}$. The average SSFR found by Brinchmann et al. (2004) is plotted as a dashed line. [See the electronic edition of the Supplement for a color version of this figure.]

to measure their stellar content (very similar results are found when using the $K$ band). Bell \& de Jong (2001) have analyzed the variation of stellar mass-to-light ratios as a function of various color indices. We have obtained the $(B-V)$ color from HyperLeda for about one-fifth of our galaxies and obtain an average value of $\langle B-V\rangle=0.6 \mathrm{mag}(\sigma=0.2 \mathrm{mag})$. This corresponds to $M / L_{H}=$ 0.57 (in solar units), and we compute the stellar mass of the galaxies in our samples using this mean $M / L_{H}$. The uncertainty is estimated to be $\sim 30 \%$ if we account for a standard dispersion of $(B-V)$ of $0.2 \mathrm{mag}$ (leading to $M / L_{H}$ falling between 0.4 and $0.8)$. The completeness in $H$ is very high in our samples ( $~ 90 \%)$, so we do not apply any corrections for the objects not detected in $H$. Xu et al. (2006) and Iglesias-Páramo et al. (2006) have discussed the relative distribution of NIR luminosities (i.e., stellar masses) within former FIR- and FUV-selected samples. Similar trends are found with the new samples, but since it is not the topic of the present work, we defer to these papers for a detailed discussion.

The SSFR distributions are shown as a function of the stellar mass in Figures 8 and 9. Once again we must account for the very different volumes sampled by our two selections, and so we accordingly calculate volume-weighted distributions to obtain the average trend of the specific SFR in the local universe. We define

$$
\langle\mathrm{SSFR}\rangle \equiv \frac{\sum_{i} \omega_{i} \mathrm{SSFR}_{i}}{\sum_{i} \omega_{i}}
$$

with $\omega_{i}=1 / V_{\max }$ for each galaxy. The calculations are all performed with our scenario 1 (upper limits treated as detections), and we calculate the geometric means and standard deviations as in $\S 4.3$ in order to be consistent with the logarithmic scales used in the study.

In Figure 8 are presented the results of the study for the FUVselected sample. The SSFR is found to decrease as the galaxy mass increases, with and without applying a volume average. This 


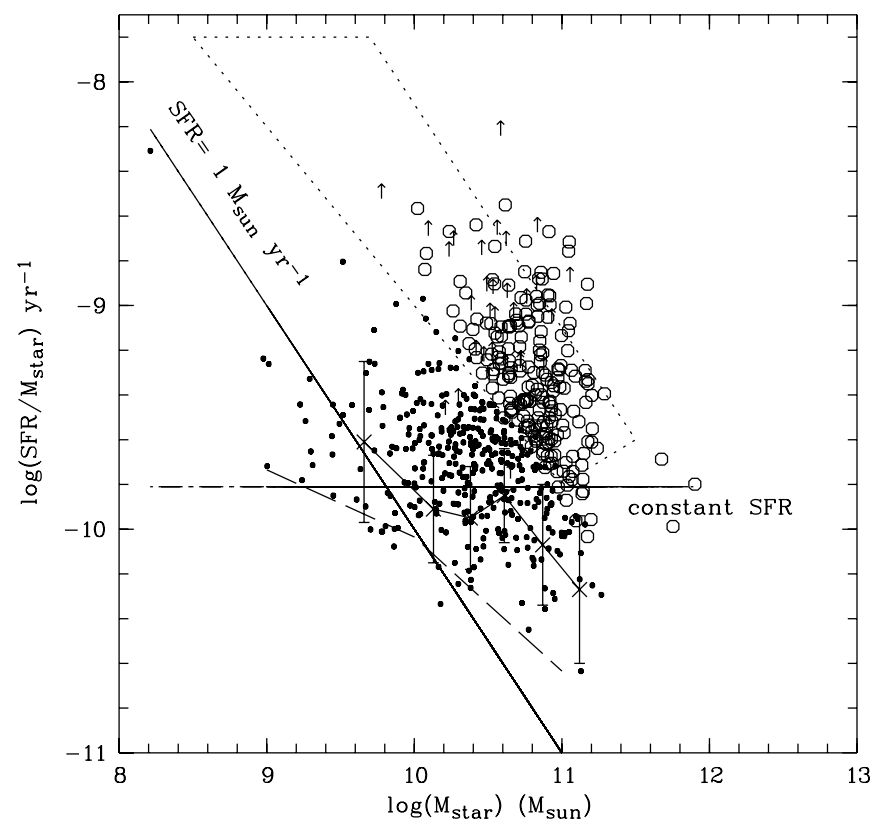

FIG. 9.-SSFR for the FIR-selected sample. The open circles represent the galaxies with $L_{\mathrm{TIR}}>10^{11} L_{\odot}$. The thin solid line shows the average SSFR, and $1 \sigma$ errors are overplotted. The horizontal line represents a constant SFR over the lifetime of the galaxy. The thick diagonal line corresponds to the present SFR equal to $1 M_{\odot} \mathrm{yr}^{-1}$. The average SSFR found by Brinchmann et al. (2004) is plotted as a dashed line. The dashed box is the locus of galaxies selected by Bell et al. (2005) at $z=0.7$. [See the electronic edition of the Supplement for a color version of this figure.]

confirms the trends found previously using optically selected samples at low and high redshifts (e.g., Cowie et al. 1996; Boselli et al. 2001; Heavens et al. 2004; Bauer et al. 2005; Feulner et al. 2005; Panter et al. 2007).

We can compare our results more precisely with similar studies of the SSFR at low redshifts. Brinchmann et al. (2004) performed a very similar analysis (which inspired our own study) based on SDSS spectroscopic data. Their result is overplotted in Figure 8, where the birthrate parameter they calculated is translated into an SSFR as given in their paper $(R=0.5$ and $t=$ 13.7 Gyr in eq. [4]). Similar trends are found; however, we obtain a larger SSFR for our galaxies selected in FUV and more massive than $\sim 10^{10} M_{\odot}$. Bauer et al. (2005) studied the evolution of the SSFR $-M_{\odot}$ relation with redshift from spectroscopic surveys of $K$-band- and $I$-band-selected samples and using the [O II] $\lambda 3727$ line to measure the SFR. We can compare our results to theirs obtained for their lowest bins in redshift $(z<0.25)$; almost all their galaxies exhibit a SSFR distribution below the diagonal line which corresponds to a SFR of $1 M_{\odot} \mathrm{yr}^{-1}$. Once again, most of our galaxies, especially with $M>10^{10} M_{\odot}$, exhibit a SFR larger than $1 M_{\odot} \mathrm{yr}^{-1}$. The spectroscopic data might be affected by aperture effects, although Brinchmann et al. (2004) developed a sophisticated method to compensate for this known effect. If we compare our results with studies based on photometric data (i.e., without aperture effects) and star formation rates deduced from the UV (rest-frame) band like that of Feulner et al. (2005), their mean value of the SSFR found at $z \sim 0.6$ and for massive galaxies $\left[10.5<\log \left(M / M_{\odot}\right)<11.5\right]$ is consistent with our results, whereas the SSFR they found for less massive galaxies at this redshift is higher than ours.

Therefore, our average values of SSFR for massive FUVselected galaxies appear larger than previously found from optical surveys at low redshifts. Several reasons might be invoked to explain this discrepancy: (1) as already suggested, the spec- troscopic surveys may suffer from aperture effects for nearby massive (and hence large-angular-sized) galaxies; (2) our FUV selection might be more biased toward galaxies forming stars actively than the optical-NIR selections; (3) our study accounts for the stellar emission reprocessed by dust in a direct way, whereas the dust attenuation in other studies is deduced from UV-optical data only. The dust attenuation is known to increase with bolometric luminosity, as does the mass of those galaxies. This parameter therefore may play a major role for the highest mass bins; (4) our derivation of the SFR assumes a correction for the dust heating by old stars (eq. [5]). A variation of $\eta$ with stellar mass may affect our results; however, to obtain values of the SSFR as low as those found by Brinchmann et al. (2004), we would have to take $\eta=0.7$ instead of 0.3 , which seems very unlikely for a galaxy sample dominated by intermediate types in the high-mass range (Sbc-Sc galaxies).

The situation appears even more complex for the FIR-selected sample, as shown in Figure 9. The sample is shifted toward more massive galaxies as compared to the FUV-selected sample (e.g., $\mathrm{Xu}$ et al. 2006). It also contains a large fraction of bright galaxies $\left(L_{\mathrm{TIR}}>10^{11} L_{\odot}\right)$, which do not follow the general trend found in FUV and optical selections: these very bright and massive galaxies exhibit a high SSFR. However, these galaxies are rare objects in the nearby universe, and as soon as a volume average is performed, we also find a global decrease of the mean SSFR when the mass increases for the FIR selection. Thus, the decrease of the SSFR as the galaxy mass increases appears to be independent of the sample selection in the nearby universe (FIR, FUV, or optical) and reflects an intrinsic mean property of the local universe. The average curve is consistent with that found for the FUV selection if we account for error bars (at the $1 \sigma$ level), but it is above the relations found by Brinchmann et al. (2004) and Bauer et al. (2005) at low $z$.

Finally, it is of some interest to compare our results with studies which also include FIR data. Caputi et al. (2006) have compared the stellar masses and star formation rates of galaxies selected at $24 \mu \mathrm{m}$ from $z=0.5$ to 3 . If we extrapolate the trend they found down to $z=0$ (in their Fig. 10), our average values of the SSFR at $z=0$ are consistent with their results. Bell et al. (2005) analyzed a rest-frame $B$-band-selected sample of galaxies at $z=0.7$ and cross-correlated it with data at $24 \mu \mathrm{m}$ (detection rate of $1 / 3$ ). The locus of the galaxies they detected at $24 \mu \mathrm{m}$ is shown in Figure 9. Their sample is complete only for galaxies more massive than $2 \times 10^{10} M_{*}$. It is clear that these galaxies are more active than the average at $z=0$ found in either the FUV or the FIR selection, thus confirming the conclusions of Bell et al. (2005). The massive galaxies detected at $z=0.7$ have a SSFR similar to what is seen in nearby LIRGs, although their morphology may well be different (Melbourne et al. 2005).

\section{CONCLUSIONS}

1. We have built large FIR- and FUV-selected galaxy samples fully representative of the nearby universe. The two selections are found to sample very different volumes, which is a direct consequence of the very different shape of the luminosity functions at the two wavelengths. Therefore, one must apply volume corrections before comparing the mean properties of these samples.

2 . The bolometric luminosity of newly formed stars is estimated by combining the infrared and ultraviolet fluxes and accounting for dust heating by old stars. The bolometric LF is calculated and found to be different in the FUV and the FIR selections. Intrinsically bright galaxies are undersampled by a FUV selection. No faint galaxy $\left(L_{\mathrm{bol}}<10^{9} L_{\odot}\right)$ is found in our FIR sample. We must wait for deeper FIR imaging surveys with a 
better spatial resolution than IRAS to compare accurately the lowest bins of the bolometric luminosity functions and investigate the contribution of close pairs and interacting systems.

3. The ratio of the total IR luminosity to the FUV $\left(L_{\mathrm{TIR}} / L_{\mathrm{FUV}}\right)$ is found to be strongly related to the TIR and to the bolometric luminosity for both samples. No universal trend is found with the FUV luminosity, making irrelevant any dust attenuation correction based on a monochromatic FUV luminosity alone. The volumeaveraged relation between $L_{\mathrm{bol}}$ and $L_{\mathrm{TIR}} / L_{\mathrm{FUV}}$ is found to be similar for both (FUV-and FIR-selected) samples for bolometric luminosities between $10^{9}$ and $5 \times 10^{10} L_{\odot}$. The monotonic increase of $L_{\mathrm{TIR}} / L_{\mathrm{FUV}}$ with $L_{\mathrm{bol}}$ continues up to $10^{12} L_{\odot}$ within the FIR selection, and $L_{\mathrm{TIR}} / L_{\mathrm{FUV}}$ saturates for FUV-selected galaxies more luminous than $5 \times 10^{10} L_{\odot}$ at a value corresponding to a dust attenuation of $\sim 2.5 \mathrm{mag}$ in the FUV.

4. The specific star formation rate is analyzed as a function of the stellar mass. It is found to decrease as the galaxy mass increases at both wavelengths and as soon as volume corrections are applied to the samples. Massive, FUV-selected galaxies and all the FIR-selected ones exhibit a larger specific star formation rate than that deduced from optical and NIR surveys of nearby galaxies with similar stellar masses. Luminous FIR-selected galaxies $\left(L_{\mathrm{bol}}>10^{11} L_{\odot}\right)$ have very large specific star formation rates, similar to those found at $z=0.7$ by Bell et al. (2005) for FIRluminous galaxies of similar mass and luminosity.

We thank the anonymous referee for her/his very useful and extensive comments. GALEX (the Galaxy Evolution Explorer) is a NASA Small Explorer, launched in 2003 April. We gratefully acknowledge NASA's support for the construction, operation, and scientific analysis for the GALEX mission, developed in cooperation with the Centre National d'Etudes Spatiales of France and the Korean Ministry of Science and Technology. V. B., D. B., and J. I.-P. gratefully acknowledge CNES and "Programme National Galaxie" support for the scientific analysis for the GALEX mission. T. T. T. has been supported by a Japan Society for the Promotion of Science Fellowship for Research Abroad for the early phase of this project, and later by the 21 st Century Center of Excellence Program "Exploring New Science by Bridging Particle-Matter Hierarchy" at Tohoku University. This research has made use of the NASA/IPAC Extragalactic Database, which is operated by the Jet Propulsion Laboratory/California Institute of Technology, under contract with the National Aeronautics and Space Administration. We also acknowledge the usage of the HyperLeda database (http://leda.univ-lyon1.fr).
Adelberger, K. L., \& Steidel, C. C. 2000, ApJ, 544, 218

Arnouts, S., et al. 2005, ApJ, 619, L43

Baldry et al. 2005, MNRAS, 358, 441

Bauer, A. E., Drory, N., Hill, G. J., \& Feulner, G. 2005, ApJ, 621, L89

Bell, E. F. 2003, ApJ, 586, 794

Bell, E. F., \& de Jong, R. S. 2001, ApJ, 550, 212

Bell, E. F., et al. 2005, ApJ, 625, 23

Boselli, A., Gavazzi, G., Donas, J., \& Scodeggio, M. 2001, AJ, 121, 753

Brinchmann, J., Charlot, S., White, S. D. M., Tremonti, C., Kauffmann, G.,

Heckman, T., \& Brinkmann, J. 2004, MNRAS, 351, 1151

Buat, V., \& Burgarella, D. 1998, A\&A, 334, 772

Buat, V., \& Xu, C. 1996, A\&A, 306, 61

Buat, V., et al. 2005, ApJ, 619, L51

Bunker, A., Stanway, E. R., Ellis, R. S., \& McMahon, R. G. 2004, MNRAS, 355,374

Burgarella, D., Le Floc'h, E., Takeuchi, T. T., Huang, J. S., Buat, V., Rieke, G. H., \& Tyler, K. D. 2008, MNRAS, in press (arXiv:0706.0810)

Burgarella, D., et al. 2006, A\&A, 450, 69

Calzetti, D., Armus, L., Bohlin, R. C., Kinney, A. L., Koornneef, J., \& StorchiBergmann, T. 2000, ApJ, 533, 682

Caputi, K. I., et al. 2006, ApJ, 637, 727

Cardelli, J. A., Clayton, G. C., \& Mathis, J. S. 1989, ApJ, 345, 245

Cortese, L., et al. 2006, ApJ, 637, 242

Cowie, L. L., Songaila, A., Hu, E. M., \& Cohen, J. G. 1996, AJ, 112, 839

Dale, D. A., Helou, G., Contursi, A., Silbermann, N. A., \& Kolhatkar, S. 2001, ApJ, 549, 215

de Lapparent, V., Arnouts, S., Galaz, G., \& Bardelli, S. 2004, A\&A, 422, 841

Feulner, G., Gabash, A., Salvato, M., Drory, N., Hopp, U., \& Bender, R. 2005, ApJ, 633, L9

Flores, H., et al. 1999, ApJ, 517, 148

Giavalisco, M., et al. 2004, ApJ, 600, L103

Gordon, K. D., Clayton, G. C., Witt, A. N., \& Misselt, K. A. 2000, ApJ, 533, 236

Heavens, A., Panter, B., Jimenez, R., \& Dunlop, J. 2004, Nature, 428, 625

Heckman, T. M., et al. 2005, ApJ, 619, L35

Hirashita, H., Buat, V., \& Inoue, A. K. 2003, A\&A, 410, 83

Hoopes, C. G., et al. 2007, ApJS, 173, 441

Hopkins, A. M., \& Beacom, J. F. 2006, ApJ, 651, 142

Hopkins, A. M., Connolly, A. J., Haarsma, D. B., \& Cram, L. E. 2001, AJ, 122, 288

Iglesias-Páramo, J., Buat, V., Donas, J., Boselli, A., \& Milliard, B. 2004, A\&A, 419,109

\section{REFERENCES}

Iglesias-Páramo, J., et al. 2006, ApJS, 164, 38

Kennicutt, R. C., Jr. 1998, ARA\&A, 36, 189

Lagache, G., Dole, H., \& Puget, J.-L. 2003, MNRAS, 338, 555

Le Floc'h, E., et al. 2005, ApJ, 632, 169

Lilly, S. J., Le Fèvre, O., Hammer, F., \& Crampton, D. 1996, ApJ, 460, L1

Lotz, J. M., et al. 2006, ApJS, submitted (astro-ph/0602088)

Martin, D. C., et al. 2005, ApJ, 619, L59

Melbourne, J., Koo, D. C., \& Le Floc'h, E. 2005, ApJ, 632, L65

Morrissey, P., et al. 2005, ApJ, 619, L7

Moshir, M., et al. 1992, Explanatory Supplement to the IRAS Faint Source Survey, Ver. 2 (JPL D-10015 8/92; Pasadena: JPL)

Ouchi, M., et al. 2004, ApJ, 611, 660

Panter, B., Jimenez, R., Heavens, A. F., \& Charlot, S. 2007, MNRAS, 378, 1550

Pérez-González, P. G., et al. 2005, ApJ, 630, 82

Reddy, N. A., et al. 2006, ApJ, 644, 792

Rowan-Robinson, M. 2001, ApJ, 549, 745

Saunders, W., et al. 2000, MNRAS, 317, 55

Schiminovich, D., et al. 2005, ApJ, 619, L47

Schlegel, D. J., Finkbeiner, D. P., \& Davis, M. 1998, ApJ, 500, 525

Seibert, M., et al. 2005, ApJ, 619, L55

Steidel, C. C., Adelberger, K. L., Giavalisco, M., Dickinson, M., \& Pettini, M. 1999, ApJ, 519, 1

Sullivan, M., Mobasher, B., Chan, B., Cram, L., Ellis, R., Treyer, M., \& Hopkins, A. 2001, ApJ, 558, 72

Takeuchi, T. T. 2000, Ap\&SS, 271, 213

Takeuchi, T. T., Buat, V., \& Burgarella, D. 2005a, A\&A, 440, L17

Takeuchi, T. T., Buat, V., Iglesias-Páramo, J., Boselli, A., \& Burgarella, D. 2005b, A\&A, 432, 423

Takeuchi, T. T., Ishii, T. T., Dole, H., Dennefeld, M., Lagache, G., \& Puget, J.-L. 2006, A\&A, 448, 525

Takeuchi, T. T., Ishii, T. T., Hirashita, H., Yoshikawa, K., Matsuhara, H., Kawara, K., \& Okuda, H. 2001a, PASJ, 53, 37

Takeuchi, T. T., Kawabe, R., Kohno, K., Nakanishi, K., Ishii, T. T., Hirashita, H., \& Yoshikawa, K. 2001b, PASP, 113, 586

Takeuchi, T. T., Yoshikawa, K., \& Ishii, T. T. 2000, ApJS, 129, 1 . 2003, ApJ, 587, L89

Wang, B., \& Heckman, T. M. 1996, ApJ, 457, 645

Wolf, C., et al. 2005, ApJ, 630, 771

Wyder, T. K., et al. 2005, ApJ, 619, L15

Xu, C. K., et al. 2006, ApJ, 646, 834

2007, ApJS, 173, 432 